\title{
Crude Oil Market Pricing Mechanism analysis and Simulation
}

\author{
Zheng Xingchen \\ Financial Research Institute of Development Research Center, Beijing, China \\ zhengxingchen@drc.gov.cn
}

\begin{abstract}
This paper aims to estimate chaos characteristics of different time in oil market and to forecast price of oil in short term. Method of Lyapunov exponent estimating and V-Statistics were utilized to estimate chaos degree and non-period cycle length, and an oil market model developed by the author was also utilized to simulate mechanism of oil price pricing process. The result shows that during the past 27 years, chaos characteristics had been reduced in oil market, but the price series enhanced its trend than before. In the case of simulation and forecasting, result shows that the oil market model can simulate the pricing process exactly. So the oil market model can be utilized to simulate the market pricing process, and forecast the price for decision making.
\end{abstract}

Key Words- chaos, market model, simulation, forecast.

\section{Introduction}

Since 2000's, international crude oil market began to climb up with large shake. According to chaos theory and fractal theory, there is a relationship between history price series. To analyze this relationship, some reports had researched chaos mechanism of crude oil market [1], and others had try to model the mechanism of crude oil market [2] [3] [4].Forecasting is important purpose of those studies. Comparing to research by chaos theory [5] [6] [7] [8], some researches which modeling and forecasting crude oil market [9] [10], had utilized regression model, such as ARCH-type models [11].

Those researches had paved the way for further researching about the mechanism of crude oil market. But there is a key problem to cope with. According to chaos theory, crude oil pricing mechanism has its orbit, but the orbit of period-doubling bifurcation has its unstable manifolds. So it is imposable to predict exact position of the orbit in long term. Even if we try to predict possible position in very short term, we still need model to simulate the pricing mechanism. But regression model can't fit chaos orbit. It is difficult to model chaos mechanism. Some researches hade utilized wavelet artificial neural network model to forecast crude oil price, but the model can $t$ explain the mechanism of oil pricing process.

This paper tries to analyze chaos characteristics of oil price series in different times, then, utilize a market model to simulate the chaos mechanism. The model was developed by the author. Data computing is supported by software MATLAB 2010 a.

\section{Chaos characteristics of price time series}

Fig. 1 shows difference of crude oil price curve. Price curve in 1980's is more plainness than which in 2000's.
First of all, the price time series should be indentified its chaos characteristics.

By the method developed by Wolf [12], we can computer the value of largest Lyapunov exponent by function (1).

$$
\lambda_{1}=\frac{1}{t_{N}-t_{0}} \sum_{k=0}^{N} \log _{2} \frac{L^{\prime}\left(t_{k}\right)}{L\left(t_{k}-1\right)}
$$

Here $\mathrm{N}$ is the length of time series, and $\lambda_{1}$ is the max Lyapunov exponent. Fig. 2 shows elements of function (1). If max Lyapunov exponent of a data series is more than zero, then the data series has chaos characteristics.

To compare chaos characteristics of price curve in 1990's and 2000's, Lyapunov exponent of price series from Jan 2, 1986 to Dec 30, 1999 was determined independently. In this period, if time-lag is set as 1, phase space was set as 5 dimension, and Lyapunov exponent is 0.0726 as in fig. 3 .

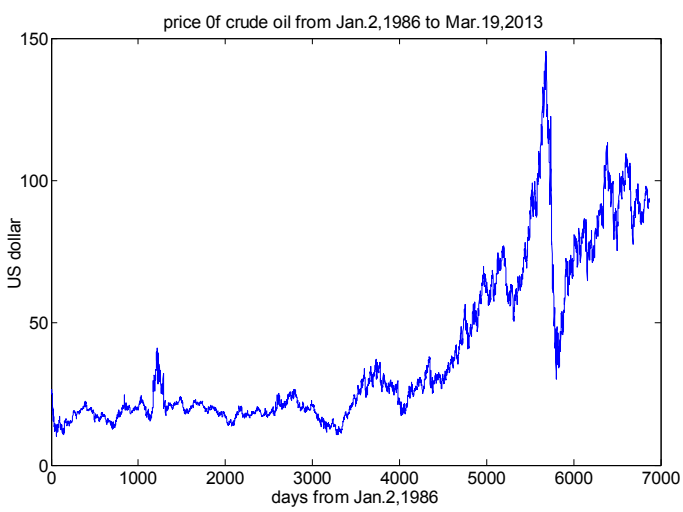

Fig.1.Crude oil price from Jan 2, 1986 to Mar 19, 2013

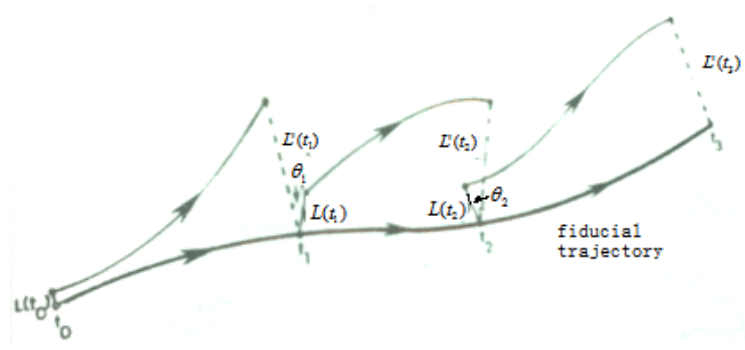

Fig.2. Method of Lyapunov exponent computing 


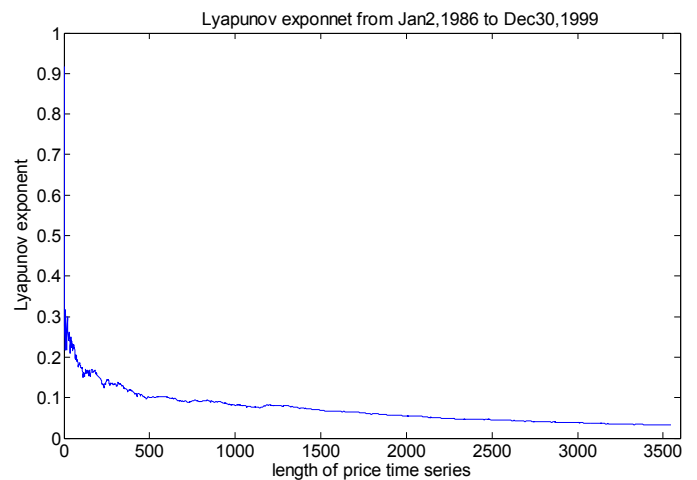

Fig.3. Lyapunov exponent from Jan 2, 1986 to Dec 30, 1999

In period from Jan 4, 2000 to Mar 19, 2013, max Lyapunov exponent is 0.0550 as in fig. 4 .

The result shows that price curve from Jan 2, 1986 to Dec 30, 1999 has more remarkable evidence of chaos characteristics.

To estimate non-period-cycle length of price series, method of V-Statistics was introduced in this case, and price series was divided into two parts as well. Method to determine value of V-Statistics was listed as function (2) to function (5).

$$
V_{n}=(R / S)_{n} / \sqrt{n}
$$

In which,

$$
\begin{aligned}
& R_{n} / S_{n}=\max \left(y_{r}\right)-\min \left(y_{r}\right) / \sqrt{\sqrt{\frac{1}{n} \sum_{r=1}^{n}\left(x_{r}-x_{m}\right)^{2}}} \\
& y_{r}=\sum_{i=1}^{r} \Delta x_{i} ; \quad r=1 \sim n \\
& x_{m}=\left(x_{1}+\cdots+x_{n}\right) / n, x_{r}(r=1 \sim n)
\end{aligned}
$$

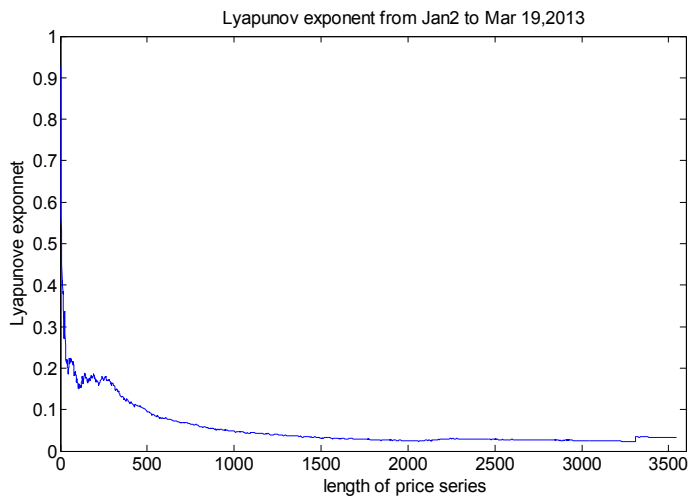

Fig.4. Lyapunov exponent from Jan 4, 2000 to Mar 19, 2013

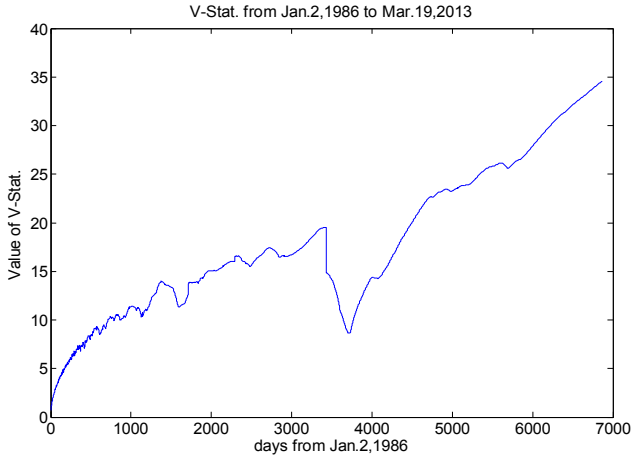

Fig.5. V-Stat. from Jan2, 1986 to Mar19, 2013

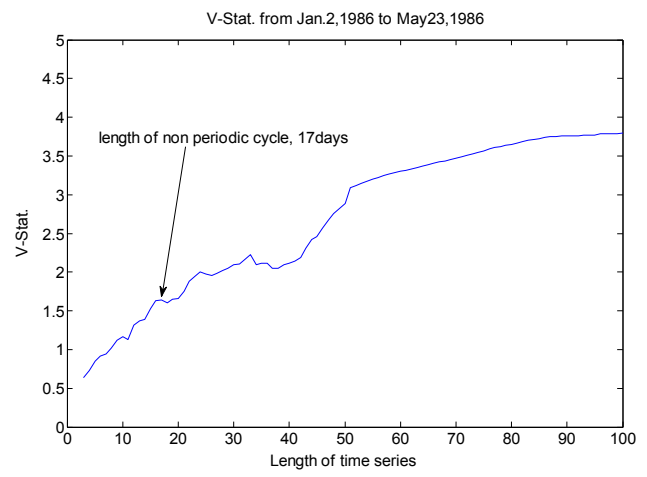

Fig.6. V-Stat. from Jan2, 1986 to May28, 1986

With the V-Statistics curve, we can indentify non-periodcycle of chaos orbit by checking turning point of the curve. According to the turning point, we can find out length of nonperiod-cycle in $\mathrm{X}$ label.

At first, total data of price series from Jan2, 1986 to Mar19, 2013 were utilized to estimate V-Statistics. In fig.5, there are many turning point located in different part of the curve.

To compare length of non-period-cycle between 1990's and 2000's, V-Statistics were estimated in small scope of 100 days. Two part of the price series was selected. One part is the early 100 daily price from Jan2, 1986 which showed in fig.6, and the other is the latest 100 daily price to Mar 19, 2013 which showed in fig.7.

By comparing two V-Statistics curve from different period, the result shows that length of non-period-cycle is similar, but the curve of V-Statistics of figure 7 turned more sharply. It means that the price series enhances its trend with more strength.

To estimate degree of data series strengthen its trend, Hurst exponent was introduced in this case.

$$
H=\left(\log \left(R_{n} / S_{n}\right)-\log (c) / \log (n)\right.
$$

Here $\mathrm{H}$ represents the Hurst exponent. If value Hurst exponent is within the zone of $(0.5,1)$, the more large of the value, the more strengthen of price series enhancing its trend. 
By utilizing price data series from Jan2, 1986 to Dec30, 1999, Value of Hurst exponent is 0.9354. And Hurst exponent of price series from Jan4, 2000 to Mar19, 2013 is 0.9882 . Comparing these two values of Hurst exponent, it is obvious that in 2000's the trend of price series was strengthened further by itself.

\section{Mechanism of simulation}

To simulate the mechanism of oil market, an oil market model by artificial intelligence theory was introduced in this case as showed in fig.8 [13] [14].

Hypothesis of the oil market model are listed as follows:

*Investor with the same appetite to risk will have the same strategy when trading.

*The decision of trading is due to the changing of gold price.

*The Prospect theory of Kahneman and Tversky is available in the model [15].

In this model, time series was rebuilt as $\mathrm{n}$ dimension in phase space. Trader was divided into $m$ kinds [16] [17].

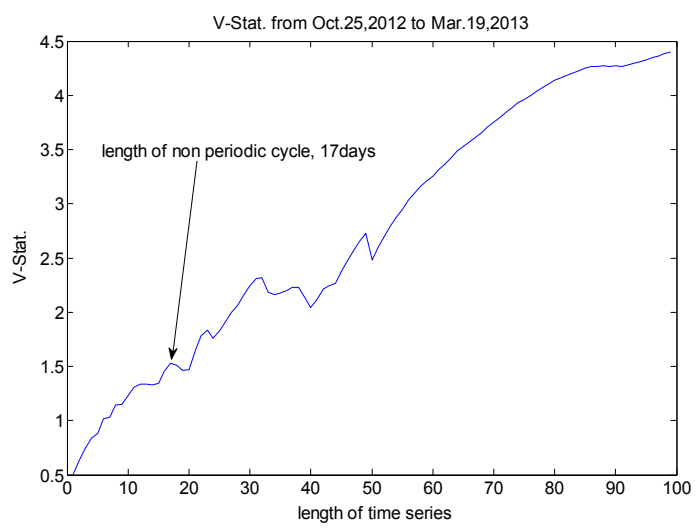

Fig.7.V-Stat. from Oct25, 2012 to Mar19, 2013

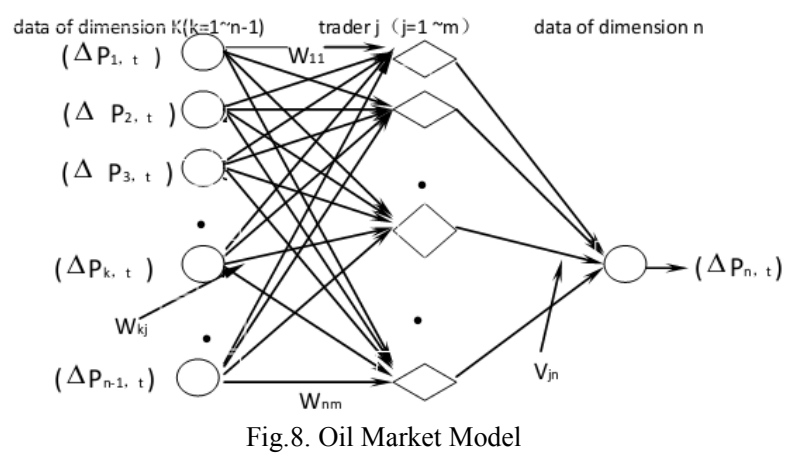

Unit $j$ of trader decision will work as fig.9.The number of $\mathrm{S}$ in fig.9 indicates the factors that trader will consider when decision. When the mechanism of the gold Market Model was conduct by fuzzy neural network, the $\mathrm{S}$ indicates the number of decision rules.

According to the Prospect theory of Kahneman and Tversky, the relationship between the subjective value and real surplus can be described by $S$ curve. Because the behavior of trader is decided by his subjective value in trading, here we assume that there is a relationship which can be described as $S$ curve between the influence to trader and real surplus. It is showed as Fig.10. The sensitivity of every trader to losing is higher than which to earning, so here the slope of curve above axis $X$ is less than which under axis $X$ in fig. 10 [18].

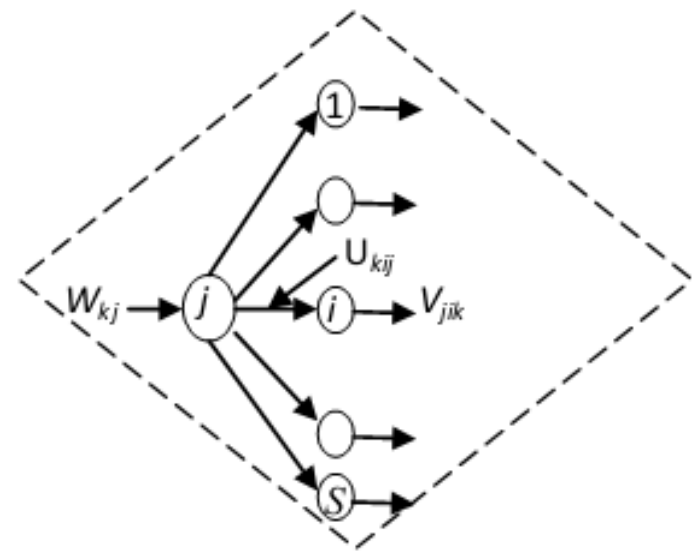

Fig.9. Structure of trader $j$

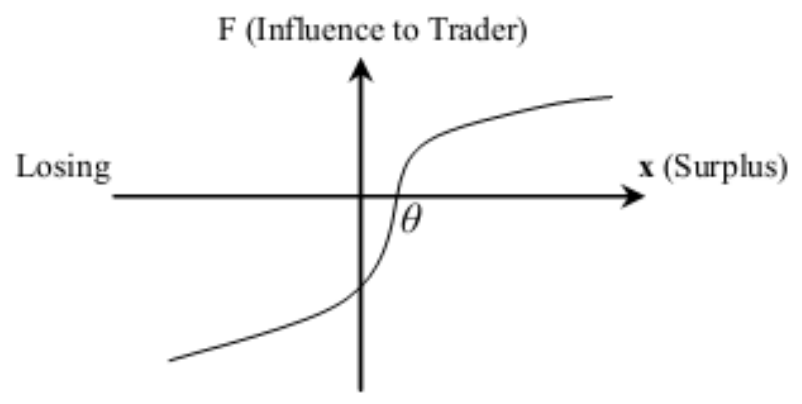

Fig.10. Relationship between Influence and Surplus

The parameter $\theta$ indicates the risk averseness of trader. The higher $\theta$ means the higher degree of risk averseness, meanwhile, the lower degree of risk appetite.

When price changing $\left(\Delta \mathrm{P}_{\mathrm{k}, \mathrm{t}}\right)$ of an asset $k$ is observed, it will influence the decision of trader $j$ according to his or her portfolio with the weight of asset $k$ express as $W_{k j}$. So the signal to trader $j$ will be:

$$
f_{k j}^{1}=W_{k j} * \Delta P_{k, t} \quad k=1, \cdots, N ; \quad j=1, \cdots, M
$$

Then, the influence of signal to trader will be:

$$
f_{k j}^{2}=\left\{\begin{array}{c}
\left.a \cdot b \cdot \tan \operatorname{sig}\left(f_{k j}^{1}-\theta\right), f_{k j}^{1}\right\rangle \theta, 0\langle a<1,0<b<1 \\
0, \quad x=\theta \\
b \cdot \tan \operatorname{sig}\left(f_{k j}^{1}-\theta\right), f_{k j}^{1}\langle\theta, 0<b<1
\end{array}\right.
$$

By the way, we can choice the value of $a$ as 0.816 .

When influenced by prices changing in financial market, the trader $j$ will make decision by his or her rule which consists of $S$ factors. To decision rule factor $i$ of trader $j$, the signal will be transformed into: 


$$
f_{j i}^{3}=\prod_{k=1}^{n} f_{k j}^{2} * U_{j i k}, \quad j=1, \cdots, M ; \quad i=1, \cdots, S
$$

In the financial market model, the number of factors influence the future pricing $\left(\Delta \mathrm{P}_{\mathrm{k}, \mathrm{t}+1}\right)$ of asset $k$ will be $\mathrm{M}^{*} \mathrm{~S}$. It means that:

$$
\Delta P_{k, t+1}=f_{k}^{4}=\sum_{i=1}^{S} \sum_{j=1}^{M} f_{j i}^{3} * V_{j i k}, \quad k=1, \cdots, N ;
$$

By the time of the model being trained, parameter, $W_{k j} U_{k i j} V_{j i k}$ and $\theta_{j}$ can be adjusted according to the function. For the asset k, the error of output will be:

$$
E_{t+1}=\frac{1}{2} \sum_{k=1}^{n}\left(\Delta P_{k, t+1}-\Delta O_{k, t+1}\right)^{2}, k=1 \sim N
$$

Here $\Delta P_{k, t+1}$ is the output of model, and $\Delta O_{k, t+1}$ is the real value of price fluctuating rate. By solving the derivative of $E_{t+1}$, we can adjust the value of parameter $W_{k j, t+1}, U_{k j, t+1}$, $V_{j i k, t+1}$ and $\theta_{j}$. When historic data of oil price were inputted into the oil market model, parameter $v_{j k}, w_{i j}$ and $\theta_{j}$ will be adjusted continually [19] [20].

\section{Result of proof-test}

To simulate the chaos mechanism of pricing process, the time-lag was set as 1 step, and phase space was rebuilt as 5 dimensions in the oil market model. Time series before Nov 14, 2012 was utilized to train the model. Daily price from Nov 14, 2012 to Nov 28, 2012 were utilized in the proof test. After being trained, the model was utilized to simulate and forecast future price. Results were listed in table below.

Table1. Results of simulation and forecasting

\begin{tabular}{|l|l|l|l|l|}
\hline & Date & $\begin{array}{l}\text { Real } \\
\text { price }\end{array}$ & $\begin{array}{l}\text { Forecasted } \\
\text { price }\end{array}$ & $\begin{array}{l}\text { Error } \\
(\%)\end{array}$ \\
\hline 1 & Nov 14, 2012 & 86.32 & 86.6531 & 0.39 \\
\hline 2 & Nov15, 2012 & 85.45 & 86.4311 & 1.15 \\
\hline 3 & Nov 16, 2012 & 86.62 & 86.6730 & 0.06 \\
\hline 4 & Nov 19, 2012 & 89.05 & 86.8022 & 2.52 \\
\hline 5 & Nov 20, 2012 & 86.46 & 86.7314 & 0.31 \\
\hline 6 & Nov 21, 2012 & 87.08 & 86.9984 & 0.09 \\
\hline 7 & Nov 23, 2012 & 87.01 & 86.5995 & -0.47 \\
\hline 8 & Nov 26, 2012 & 87.28 & 87.0309 & -0.29 \\
\hline 9 & Nov 27, 2012 & 86.81 & 87.9928 & 1.36 \\
\hline 10 & Nov 28, 2012 & 86.10 & 87.3265 & 1.42 \\
\hline
\end{tabular}

In this table of results, $90 \%$ of forecasted price have their error less than $1.5 \%, 60 \%$ of forecasted price have their error less than $0.5 \%$. It proves that the oil market model has its strength in simulation and forecast.

\section{Conclusion}

This research concentrated in oil market chaos characteristics analysis and pricing mechanism simulation. Results of chaos analysis indicate that the crude oil market has experienced a period in which chaos index reduced, but price fluctuation was enhanced. It suggests that price analysis and forecast are needed to avoid risk in market.

On the other hand, proof-test shows that the oil market model has high capability in simulation and forecast. It also proved that the chaos mechanism of oil market can be simulated forecasted in short term.

As a new method to simulate and forecast chaos pricing mechanism, the oil market model can be utilized in research and decision making.

\section{Reference}

[1] Bahram Adrangi, Arjun Chatrath, Kanwalroop Kathy Dhanda, Kambiz Raffiee. Chaos is in oil prices? Evidence from futures markets, Energy Economics, 2001(23): 405-425.

[2] Rongbao Gu, Hongtao Chen, Yudong Wang. Multi fractal analysis on international crude oil markets based on the multi fractal detrended fluctuation analysis, Physica A: Statistical Mechanics and its Applications, 2010(389): 2805-2815.

[3] Jose Alvarez-Ramirez, Eduardo Rodriguez, Esteban Martina, Carlos Ibarra-Valdez. Cyclical behavior of crude oil markets and economic recessions in the period 1986-2010, Technological Forecasting and Social Change, 2012(79): 47-58.

[4] Saskiater Ellen, Remco C.J. Zwinkels. Oil price dynamics: A behavioral finance approach with heterogeneous agents , Energy Economics, 2010(32): 1427-1434.

[5] Sang Hoon Kang, Sang-Mok Kang, Seong-Min Yoon. Forecasting volatility of crude oil markets , Energy Economics, 2009(31): 119-125.

[6] F.Gori, D.Ludovisi, P.F. Cerritelli. Forecast of oil price and consumption in the short term under three scenarios: Parabolic, linear and chaotic behavior, Energy, 2007(32)1291-1296.

[7] Shahriar Yousefi, Ilona Weinreich, Dominik Reinarz. Wavelet-based prediction of oil prices, Chaos, Solitons \& Fractals, 2005(25): 265-275.

[8] Jose Alvarez-Ramirez, Jesus Alvarez, Eduardo Rodriguez. Short-term predictability of crude oil markets: A detrended fluctuation analysis approach, Energy Economics, 2008(30:2645-2656.

[9] Antonia Azzini, Célia da Costa Pereira, Andrea G. B. Tettamanzi. Modeling Turning Points in Financial Markets with Soft Computing Techniques, Natural Computing in Computational Finance Studies in Computational Intelligence, 2010 (293):147-167.

[10] Rania Jammazi, Chaker Aloui. Crude oil price forecasting: Experimental evidence from wavelet decomposition and neural network modeling, Energy Economics, 2012(34):828-841.

[11] Chin Wen Cheong. Modeling and forecasting crude oil markets using ARCH-type models, Energy Policy, 2009(37):2346-2355.

[12] Wolf, A. Swift, "Determining Lyapunov Exponents from a Time Series,"Physic 16D, July 1985.

[13] R.G. Palmer, W.B. Arthur, J.H. Holland, B. LeBaron, Artificial economic life: a simple model of a stock market, Physica D, 1994 (75) 264-274.

[14] A. Ponzi, Y. Aizawa: Evolutionary financial market models , Physica A, 2000(287): 507-523.

[15] Kahneman D, Tversky A. Prospect theory: An analysis of decision under risk, Econometrica, 1979(47): 263-291.

[16] Cao L. Practical method for determining the minimum embedding dimension of a scalar time series, Physica D, 1997,110(12): 43-50.

[17] Scheinkman J.A, Le Baron B. Nonlinear dynamics and stock returns, Journal of Business, 1989, 62(3): 311-337.

[18] Barberis Nicholas, Andrei Shleifer, Robert W.A model of investor sentiment, Journal of Economics, 1998, 49(3): 307-343.

[19] Granger,C.W.J.\& Tersvirta.T.(1993). Modelling Nonlinear Economic Relationships, Oxford University press, Oxford.

[20] Holland J. Adaption in Natural and Artificial Systems: An Introductory Analysis with Application to Biology, Control and Artificial Intelligence. 2ndEdition. Cambridge: MIT Press/Bradford Books, 1992. 